\title{
Eisenchelation verbessert das Blutbild
}

\section{Chronische Transfusionsbedürftigkeit ist mit einem hohen Risiko der Eisenüberladung des Organismus verbunden, quantifizierbar anhand des Serumferritinwerts. Wie aus der 2010 publizierten EPIC-Studie hervorgeht, senkt eine 12-monatige Therapie mit dem Eisenchelatbildner Deferasirox (Exjade ${ }^{\circledast}$ ) den Serumferritinspiegel bei transfusionsbedürftigen Patienten mit myeloischen Dyspla- sien (MDS) signifikant.}

Erstmals wies eine aktuelle PostHoc-Analyse dieser Subgruppe von Patienten auch eine Blutbildverbesserung unter Eisenchelation, betonte Norbert Gattermann, Düsseldorf.

In dieser von Gattermann geleiteten Analyse wurden die 341 Patienten beobachtet, die in der MDS-Subgruppe der EPIC-Studie eingeschlossen waren. Endpunkte waren das erythroide, thrombozytäre und neutrophile Ansprechen während einer einjährigen Deferasirox-Therapie. Die Responderraten für diese drei Parameter betrugen 22,6\%, $14,0 \%$ und $19,6 \%$. Wie aus der Veränderung des Serumferritins bei hämatologischen Respondern und Non-Respondern hervorgeht, ist die Eisenentfernung ursächlich für die Blutbildverbesserung, so Gattermann. Besserung der hä- matologischen Parameter, einhergehend mit einer verminderten Transfusionsbedürftigkeit seien vor allem bei Patienten erreicht worden, deren Serumferritin deutlich abnahm.

Als wahrscheinlichste Ursache dafür vermutet Gattermann eine Verminderung des oxidativen Stress unter Eisenchelation. Auch seien spezielle pharmakologische Effekte von Deferasirox wie die Hemmung von NFkB nicht auszuschließen. Zur Erhärtung dieser Ergebnisse seien prospektive klinische Studien unerlässlich, betonte Gattermann. zi

Round Table Discussion am 25. Januar 2011 in Düsseldorf; Veranstalter: Novartis Oncology

\section{Chronische Myeloische Leukämie (CML) Nilotinib übertrifft Imatinib in der First-Line weiterhin}

\begin{abstract}
Eine Reihe von Studien, die auf der Jahrestagung der American Society of Hematology (ASH) im Dezember vorgestellt wurden bestätigen die Vorteile der Nilotinib-Therapie gegenüber der Behandlung mit Imatinib, dem Tyrosinkinasehemmer der ersten Generation.
\end{abstract}

Die 24-Monatsdaten der ENESTnd-Studie belegen die Überlegenheit von Nilotinib gegenüber Imatinib bei neu diagnostizierten Patienten in der chronischen Phase der CML [Hughes T et al. Blood. 2010;116: Abstract \#207]

In der zulassungsrelevanten Studie wurde Nilotinib in zwei Dosierungen $(2 \times 300 \mathrm{mg} / \mathrm{Tag}$ oder $2 \times 400 \mathrm{mg} / \mathrm{Tag}) \mathrm{mit}$ dem bisherigen Standard Imatinib (1 x $400 \mathrm{mg} / \mathrm{Tag}$ ) verglichen: Nilotinib zeigt ein schnelleres und tieferes Ansprechen auf molekularer und zytogenetischer Ebene. So betrug die mediane Dauer bis zum Erreichen einer guten molekularen Remission (MMR) 6 bzw. 8 Monate unter Nilotinib und 10 Monate unter Imatinib [Hochhaus A et al. Blood. 2010;116: Abstract \#3431]. Sowohl die MMR-Rate nach 24 Monaten als auch die Rate der kompletten molekularen Remission (CMR) waren in den NilotinibGruppen signifikant günstiger (62 bzw. 59 vs. $37 \%$; $<<0,0001$, reps. 26 bzw. 21 vs. $10 \%$ ( $<<0,0001$ bzw. $\mathrm{p}=0,004)$. Ein komplettes zytogenetisches Ansprechen (CCyR) wurde bei 87 bzw. $85 \%$ der Patienten in den Nilotinib-Gruppen erreicht $(\mathrm{p}=0,0018)$, in der Imatinib-Gruppe bei $77 \%(\mathrm{p}=0,016)$.

Dieses gute Ansprechen wird auch durch andere Studien [ Coneally E et al. Blood 2010;116: Abstract \#3427 und Rosti G et al. Blood 2010;116: Abstract \#359] bestätigt und spiegelt sich in einer geringen Krankheitsprogression wider: Nach 24 Monaten waren unter Nilotinib zwei $(2$ x $300 \mathrm{mg} /$ Tag) bzw. drei (2 x $400 \mathrm{mg} / \mathrm{Tag})$ Patienten progredient,während unter Imatinib bei 12 Patienten ein Progress in die akzelerierte Phase oder in die Blastenkrise beobachtet werden konnte.

Auch die Sicherheit und Verträglichkeit von Nilotinib wurde im Rahmen der ENENSTndStudie untersucht: So zeigten beispielsweise Patienten mit vorbestehendem Typ-2-Diabetes bei Wirksamkeit und Vertraglichkeit ähnliche Ergebnisse wie die Gesamtpopulation: Selbst Nilotinib-assoziierte Hyperglykämien führten bei diesen Patienten nicht zur Unterbrechung der Therapie [Saglio G et al. Blood. 116: Abstract \#3430]

db

Nach Information von Novartis Oncology 